\title{
Fatores ambientais associados Às helmintoses INTESTINAIS EM ÁREAS DE ASSENTAMENTO SUBNORMAL, JUIZ DE FORA, MG*
}

\author{
EN VIRON MENTAL FACTORS RELATED TO INTESTIN AL HELMINTH \\ INFECTIONS IN SUBNORMAL SETTLEd AREAS, JUIZ dE Fora, MG*
}

\section{JúLO CÉSART EIXEIRA}

Engenheiro Civil edeSegurançano Trabalho. D outor em Saneamento, M eio Ambientee Recursos H ídricos. Professor Adjunto do D epartamento deH idráulica eSaneamento daU niversidade Federal deJ uiz deFora

\section{Léo HeLler}

Engenheiro C ivil. D outor em Epidemiologia. Professor Adjunto do D epartamento deEngenhariaSanitáriae Ambiental daU niversidadeF ederal deM inas $G$ erais

\author{
Recebido: 26/12/03 Aceito: 09/06/04
}

\section{RESUMO}

O sobjetivos destetrabal ho são o decaracterizar a morbidadepor infecções intestinais por helmintos eo de identificar os fatores associados a estas doenças, com ênfase para os fatores ambientais, em crianças com idade entre um ano completo e cinco anos incompletos residentes em áreas de assentamento subnormal áreas deinvasão. U tilizou-seum delineamento epidemiológico transversal de base populacional em 29 das 78 áreas de assentamento subnormal do município de uiz deFora- M G. A amostra foi composta por todas as criançasna fai xa etária de interesse residentes nos 29 assentamentos, escolhidos deforma não aleatória. A amostra estudada totalizou 753 crianças. A coleta de dadosfoi feita medianteentrevistas domiciliarescom a mãe ou 0 responsável pela criança. U tilizou-se o método de H offmannPons-J aner no exame parasitológico de fezes. Foram usados modelos de regressão logística binária paraidentificar fatoresassociados às enfermidades. D a amostra, $161(21,38 \%)$ criançasapresentaram infecções intestinais spor helmintos. 0 sfatores associa dosa estas parasitoses incluíram idade da criança, rendafamiliar, queixas quanto à qualidade da água do sistema público edeficiências no esgotamento sanitário.

PALAVRAS-CHAVE: I Ifecções intestinais por hel mintos, saneamento ambiental, saúdeambiental, parasitas, saúdeinfantil (saúdepública).

\section{ABSTRACT}

Theobjectives of thisstudy arethecharacterization of themorbidity byintestinal helminth infectionsand theidentification of thefactors associated with thesediseases, with emphasison environmental factors, in children ranging from oneto fiveincompleteyearsold residing in subnormal settlement areas- invasion areas. A population-based cross-sectional epidemi ological design wasused in 29 out of the 78 subnormal settlement areas in the municipality of J uiz de F ora, M G. Thesampleconsisted of all children at theageof interest residing in the 29 settlement areas, not chosen at random. Theassesed sample amounted to 753 children. D ata were collected by means of domiciliary interviews with their mothers or with the person responsiblefor them. TheH offmann-PonsJaner method wasused in theparasitological fecesexamination. Binarylogisicregression models were used to identify the factors associated with the diseases 0 ne hundred, sixty and one (21.38\%) sample children presented intestinal helminth infections. The factors associated with these parasitic diseases included the children's age, family income, complaintsabout thequality of thewater from thepublic system and deficienciesin sanitation.

KEYWORDS: Intestinal helminth infections, environmental sanitation, environmental health, parasites, child health (public health).

\section{INTRODUÇÃO}

O sparasitas intestinais estão entre os patógenosmaisfreqüentementeencontrados em seres humanos. D iversos fatoresinfluem no sentido de que exista esta situação, massaneamento ambiental ausente ou deficiente, práticas de higiene inadequadas e condições precárias nas quaisvivem milhões de pessoas constituem os maisimportantes.

A O rganização M undial deSaúde estimou, em 1997, queexistiam, em todo o mundo, cerca deum bilhão de indivíduosinfectad os por Ascarislumbricoi des, entre 800 e 900 milhões albergando Trichuris trichiura e ancilostomídeos, 400 milhões infectados por Entamoeba histolytica e 200 milhões por Giardia lamblia (W H O, 1997).

O s danos que os parasitas intestinais podem causar a seus portadores incluem, entre outros agravos, a obstrução intestinal (Ascarislumbricoides), a desnutrição (Ascaris lumbricoides e Trichuris trichiura), a anemia por deficiência de ferro (ancilostomídeos) e quadros de

* Trabalho subvencionado pelo Conselho N acional de D esenvolvimento Científico eTecnológico (C N Pq - Processo n 476.900/2001-8) e pela C oordenação de Aperfeiçoamento de Pessoal deN ível Superior (CAPES/U FJF - Processo n².573/2001-8). 
diarréia e má absorção de nutrientes (Entamoeba hisolytica eGiardia lamblia), sendo queasmanifestaçõesclínicassão usualmente proporcionais à carga parasitária albergadapelo indivíduo (Stephenson, 1987).

A relação entreo ambienteeatrans missão de parasitoses foi estudada, entre outros, por M ara \& Feachem (1999), que propuseram uma classificação ambiental unitária de doenças relacionadasàágua e aos esgotos, composta por sete categorias dedoenças. N essa classificação, a categoria "geohelmintoses" incluiu a presença nas fezes de ovos e/ ou larvas de Ascaris lumbricoides, Trichuris trichiura, Strongyloidestercoraliseancilostomídeos.

EmJuiz deFora- M G (PJF, 2001), cerca de 78 áreas, alvo de invasões, em processo de legalização ou não, abrigavam aproximadamente 32 mil pessoasem 8,4 mil moradias, no ano de 2001. Isto significa quepelo menos $7,0 \%$ da população da cidade viviam em situação precária. D adosdo C enso de 1991 do IBGE (FIBGE, 1991) mostravam queo número de assentamentos de submoradias no município era 50. Logo, taisfontesindicavam que, no período 1991-2000, o número de pessoas em áreas de assentamento subnormal no município - áreas de invasão - cresceu 3,1 vezes mais do quea população detodaa cidade, respectivamente $5,88 \%$ e $1,89 \%$ ao ano.

Como em todo o país, a maioria dos moradores de áreas de habitação subnormal édesempregada, subempregada ou migrante de cidades menores, que vêem, em cidades degrande ou mé dio porte, a chancedeuma vidamelhor. Assim, frenteà falta deemprego ederenda, o problema de ocupação de assentamentossubnormais vem seagravando nos últimosanos, expondo milhões debrasileiros a condições de vida precárias, principalmenteem relação à habitação, ao saneamento eàs condições dehigiene.

A relevância desta constatação foi reforçada com os resultados do Censo 2000 no Brasil, que revelaram o grande crescimento das cidades degrandeemé dio porte, com índices crescentes de favelização, de desemprego, demisériae, provavelmente, deparasitosesintestinais.

D estemodo, 0 objetivo desteestudo foi caracterizar as infecções intestinais por helmintos, identificar os fatoresassociadosa estas doenças, com ênfasepara os fatoresambientais, em crianças com idade entreum ano completo ecinco anos incompletosresidentesem áreas de assentamento subnormal - áreas deinvasão visando contribuir para queasinterven- ções em saneamento e saúde possam ser priorizadas deforma mais eficaz, no sentido de proteger a saúde infantil.

\section{METODOLOGIA}

\section{Área geográfica abrangida}

A sel eção dasáreas deestudo foi re alizada após contato com órgãos da Prefeitura deJuiz deFora- M G: aCompanhia de Saneamento M unicipal eaEmpresa Regional deH abitação. 0 rganizações não governamentaisligadasà I greja C atólica e ao movimento de defesa dos direitos humanostambém foram consultadas. Considerando as exposições a estudar, 29 assentamentos, com aproximadamente 2.700 moradias e população estimada em 12.000 habitantes, foram selecionados, deforma não al eatória, para compor a amostra de estudo.

A escolha dosassentamentos deforma não aleatória foi adotada em virtude da necessidade de se ter na amostra comunidadescom diferentestipos desoluções para suas necessidades sanitárias como, por exemplo, comunidades com abastecimento deágua do sistema público - aindaqueclandestino - , comunidades abastecidas por água de caminhõespipa, comunidades abastecidas com água de poços freáticose, ainda, comunidades que utilizavam água de minas e/ou de córregosin natura para consumo humano.

\section{Delineamento epidemiológico}

0 método epidemiológico empregado consistiu deum estudo transversal, também denominado deestudo seccional ou deprevalência. Pode ser melhor compreendido como o estudo epidemiológico no qual fator(es) eefeito(s) são observados num mesmo momento.

\section{Amostra}

$N$ esteestudo, o dimensionamento do tamanho daamostrafoi obtido por meio do softwareEPI IN FO 6.0, apartirdeprevalências esperadasparadiferentesparasitasintestinais obtidasjunto àliteratura. Admitiu-se:

- $\mathrm{N}$ ível designificância =5\%;

- Poder estatístico do teste $=80 \%$;

- Reação não-expostos/expostos =1:1;

- Prevalência esperada entreos expostos:

- Ascaris lumbricoides $=66,4 \%$ (M oraes, 1997);
- Trichuris trichiura $=87,8 \%$ (M oraes, 1997);

- Ancilostomídeos = 25,2\%

(M oraes, 1997).

- Prevalênciaesperadaentreosnãoexpostos:

- Ascaris lumbricoides = 38,0\% (M oraes, 1997);

- Trichuris trichiura $=68,1 \%$ (M oraes, 1997);

- Ancilostomídeos = 9,4\% (M oraes, 1997).

Assim, o tamanho da amostra foi estimado em, no mínimo, 650 crianças com idadeentreum ano completo ecinco anos incompletos, por ser esta faixa etária mais suscetível às geohelmintoses. É importante destacar que, em cada habitação visitada, onde existiam crianças nafaixa etária deinteresse, todas estas crianças foram convidadas a fazer o exame parasitológico defezes.

\section{Entrevistas domiciliares e exames de fezes}

Foram aplicados 659 protocolos de entrevista nos 29 assentamentos objeto de estudo. As entrevistasforam real izadas por equipetreinada especificamentepara estefim, recrutada entreentrevistadores quetrabalharam no $C$ enso brasileiro de 2000 na cidade de Juiz de Fora - M G. Foram obtidas fezes de 753 crianças - a saber, material biológico das crianças alvo das entrevistas e de alguns de seus irmãos - , cujos responsáveis coletaram material biológico para o exame parasitológico defezes.

A pós a coleta de fezes em todas as moradias visitadas no dia anterior ou na antevéspera, 0 entrevistador dirigia-se imediatamentea um laboratório demedicina ambulatorial, certificado com ISO 9002, para a entrega do material biológico. 0 laboratório utilizou o método deH offmann-Pons-J aner (H offmann, Pons \& Janer, 1934) no exame parasitológico defezes.

Portanto, o tamanho final daamostra obtida foi de 753 crianças.

\section{Definições para "Doentes" e "Não-Doentes"}

\section{i) Doentes}

Criança selecionada, com idadeentreum ano completo ecinco anosincompletos, residentena área geográfica deestudo, em cujas fezestenham sido identificados ovos e/ou larvas de Ascaris 
lumbricoides, Trichuris trichiura, Strongyloides stercoralis ou ancilostomídeos.

\section{ii) Não-dbantes}

Criança selecionada, com idadeentreum ano completo ecinco anosincompletos, residentenaárea geográfica de estudo, em cujas fezes não tenham sido identificados ovos e/ ou larvas de Ascaris lumbricoides, Trichuris trichiura, Strongyloides stercoralis ou ancilostomídeos.

\section{Análise estatística}

A análise dos dados foi feita por meio do software Statistical Package for theSocial Sciences(SPSS), versão 10.0.

Fez-se inicialmente uma análise exploratória dos dados, por meio de tabelas defreqüênciassimples, além daaná lise univariada com medidas de associação etestes designificância. Posteriormente, aplicou-se a técnica de regressão logística binária a um grupo de 25 variáveis independentes, utilizando-se o mé todo stepwise backward, de forma manual, para eliminação devariávésnão significativas, visando identificar a associação entreas variáveis independenteseas doenças estudadas. Para análise dos dadosfoi adotado o oddsratio- OR.

A análisemultivariadadosdadosfoi desenvolvida por meio de um processo evolutivo, em etapas seqüenciais, deformaa permitir a eliminação progressivadas exposições não associadas às doenças. Tal processo envolveu asseguintes atividades:

1. Seleção preliminar de variáveis com significância inferior a 0,25 $(p<0,25)$ paraintegrar o modelo inicial;

2. Análise das variáveis préselecionadas segundo oito subgrupos explicativos;

3. Eliminação de variáveis dos subgrupos que apresentaram uma significância superior a $0,15(p>0,15)$;

4. Construção do modelo final com as variáveis remanescentes em cada um dos oito subgrupos, para o grupo de doenças estudadas, com a manutenção de variáveis que apresentaram uma significância inferior a 0,05 $(p<0,05)$.

\section{Ética}

O ComitêdeÉtica em Pesquisas da U FM G - COEP, segundo parecer específico datado de 24 de abril de 2002 , aprovou o presente estudo, estando os procedimentos deacordo com os padrões éticos de experimentos com humanose de acordo com a D eclaração deH elsinki de 1964, reformulada em 1975, 1983, 1989, 1996 e 2000 e, ainda, de acordo com a Resolução 196/96 do Conselho $\mathrm{N}$ acional deSaúde do Brasil.

\section{RESULTADOS}

\section{Caracterização das helmintoses intestinais}

D a amostra de 753 crianças, 161 (21,38\%) apresentaram helmintoses. As prevalênciasforam de $14,74 \%, 11,02 \%$, $1,99 \%$ e $1,33 \%$, respectivamente para Ascarislumbricoides, Trichuristrichiura, ancilostomídeos eStrongyloidesstercoralis

\section{Fatores de risco para infecções intestinais por helmintos}

N a análise multivariada, 25 das 37 variáveis estudadas constituíram o modelo inicial $(p<0,25$ na análise univariada), sendo:

- Estruturafamiliar $=6$ variáveis;

- N ível sócio-econômico =7 variáveis;

- H ábitoshigiênicos =1 variável;

- Abastecimento de água = 3 variáveis;

- Esgotamento sanitário =3 variáveis; variavess

- M anejo de resíduos sólidos =3

- D renagem pluvial =1 variável;

- Presença devetores =1 variável.

$\mathrm{No}$ ajuste final do modelo multivariado, as variáveis mostradas na Tabela 1 apresentaram associação estatisticamentesignificativa com as infecções intestinais por helmintos, segundo osrespectivosodds ratio:

\section{DISCUSSÃO}

0 model o final obtido pela análise multivariada para as infecções intestinais por helmintos-Tabela 1 - revelou que variávesis relacionadasà estrutura familiar (idadedacriança), às condiç̧ões sócio-econômicas (renda familiar) evariáveisrelacionadas ao ambienteondevivea criança (queixas quanto à qualidade da água e deficiências no esgotamento sanitário) mostraram-sefatores associados àsenfermidades estudadas.

$\mathrm{U} \mathrm{m}$ dos fatores associados às helmintosesintestinaiséaidadeda crian- ça, que apresentou um caráter de risco com $0 \mathrm{R}$ de 1,226. Viu-se que quanto maior a idadeda criança, maior o risco da mesma apresentar parasitoses intestinais. Este achado é explicado pelo fato de as crianças mais vel has deslocarem-se por toda a área do assentamento subnormal, muitas vezes descalças, o queaumentaem muito o risco de se infectarem com helmintos pelo contato da pelecom o solo contaminado.

A rendafamiliar consisteem um fa tor deproteção para ashelmintosesintes tinais, com O R apresentando um gradiente decrescente à medida que a renda familiar aumenta, como era esperado. Tal achado confirma serem as helmintoses intestinais uma doença característica da camada mais pobre da população.

Em relação aosfatores ambientais associados às helmintoses intestinais, pode-seafirmar que, quanto à queixa sobre a água da rede pública de abastecimento, por meio da comparação dicotômica "sim" e "não" encontrou-seum caráter derisco à saúde para estas doenças ( $O R=2,073)$. N esta pesquisa, a queixa quanto à qual idade da água estava, normalmente, associada à sua turbidez, ou seja, à presença de sólidos em suspensão naágua. A turbidez naágua podeter le vado a doismecanismos de contaminação das crianças estudadas. Em um primeiro mecanismo de transmissão, a turbidez na água pode ter levado a comunidadeautilizar fontesalternativas de abastecimento de água sem turbidez, a saber, - como observado nos trabalhos de campo -, água demina ou denascente, e água de poços rasos; portanto, a ingestão de água de fontes alternativas pode ter contribuído para o parasitismo por Ascaris lumbricoidesepor Trichuristrichiura, que, embora sejam geohelmintos, também estão associados ao consumo de água imprópria para a saúde. Em um segundo mecanismo detransmissão, a turbidez da água pode ter servido de abrigo para os microrganismos patogênicos citados, dificultando uma desinfecção eficaz nas Estações deTratamento deÁ gua (H eller $\&$ Casseb, 1995; AW WA, 1999).

Ainda, em relação à queixa quanto à qualidade da água de abastecimento, M ara \& Feachem (1999) destacam a importância do aspecto estético agradável das águas deabastecimento, deforma a evitar o consumo de água contaminada. 0 sautores afirmam que o consumo de água de fontes alternativas facilita a infecção por parasitasintestinais por meio da ingestão da própria água, de vegetais 
Teixeira, J. C. \& Heller, L.

Tabela 1 - H elmintoses intestinais - análise multivariada estimativas pontuais de $\mathrm{O} \mathrm{R}$ e intervalo de confiança a $95 \%$

\begin{tabular}{|c|c|c|c|}
\hline Exposiçãao & Categorias & OR (IC a 95\%) & $\mathrm{p}$ \\
\hline Idade da criança & - variável contínua & $1,226(1,022-1,471)$ & 0,028 \\
\hline Renda familiar* & $-\geq 1$ s.m. e $<2$ s.m. & $0,571(0,334-0,977)$ & 0,041 \\
\hline Renda familiar* & $-\geq 2$ s.m. e $<3$ s.m. & $0,363(0,157-0,884)$ & 0,019 \\
\hline Renda familiar* & $-\geq 3$ s.m. & $0,258(0,082-0,807)$ & 0,020 \\
\hline Queixa sobre a água** & $-\operatorname{sim}$ & $2,073(1,037-4,143)$ & 0,039 \\
\hline Disposição dos esgotos*** & - em algum tipo de fossa & $0,244(0,114-0,523)$ & $<0,001$ \\
\hline Disposição dos esgotos*** & - em córregos & $0,370(0,173-0,791)$ & 0,010 \\
\hline Disposição dos esgotos*** & - rua ou terreno & $3,474(1,599-7,548)$ & 0,002 \\
\hline \multicolumn{4}{|c|}{$\left(^{*}\right)$ s.m. $=$ salário mínimo no Brasil $=\mathrm{R} \$ 240,00 \approx \mathrm{US} \$ 80,00$} \\
\hline \multicolumn{4}{|c|}{$\left(^{*}\right)$ categoria de comparação = menos de um salário-mínimo } \\
\hline \multicolumn{4}{|c|}{$\left({ }^{* *}\right)$ categoria de comparação $=$ não } \\
\hline$\left({ }^{* * *}\right)$ categoria de compara & coletora & & \\
\hline
\end{tabular}

cruse de partículas deterra contaminados, por exemplo, com Ascarislumbricoides eTrichuristrichiura.

Q uanto ao esgotamento sanitário, háevidências dequeo maior risco para as helmintoses intestinais está associado à disposição dosesgotosno terreno ou nas $\operatorname{ruas}(O R=3,474)$.

Em relação à associação entrea disposição deesgotosno terreno ou na ruae helmintoses intestinais, Azevedo (2003) encontrou uma razão de preval ências de 1,88 , quando comparou um grupo que lançava os esgotos no terreno com outro que dispunha as excretas em redes coletoras. M oraes (1996), estudando as helmintoses em criançascom idadeentre cinco equatorzeanos, encontrou valores de odds ratio variando de 2,59 a 2,78, concluindo que há associação estatisticamentesignificativa entre disposição inadequada de esgotos no terreno e a prevalência de helmintoses intestinais $(p \varangle 0,0001)$. Feachem et al. (1983) destacam que os geohelmintos têm longos períodos de latência, são persistentesno ambiente e medidas de disposição de excretas têm um papel mais importante nalimitação dosmecanismos desuatransmissão do queas práticas dehigiene. Dando consistênciaatodosetesachados, aW H O (1991) concluiu queadisposição inadequada deexcretaslevaàdisseminação degeohelmintos, particularmentedeAscarislumbricaides Trichuris trichiura eancilostomídeos.
D eve-sedestacar que asfossas, nesta pesquisa, demonstraram constituir-seem uma solução eficaz para a disposição dos esgotos, uma vez que apresentaram caráter deproteção em relação àshelmintoses intestinais $(O R=0,244)$. A hipótesemais consistente é de que nas redes coletoras construídas, muitas vezes, em regimede mutirão pela própria comunidade, verificam-seinúmeros problemas, como deficiência nas ligações entre tubos, redes perfuradas com inúmeros pontos devazamento e, até, devido à pouca profundidade de assentamento, redestotalmente seccionadas. Por outro lado, deve-se destacar queo uso defossas, quando bem construídas emantidas, constituem em uma solução sanitariamente adequada para o destino dos dejetos sanitários (von Sperling, Costa \& Castro, 1995). D este modo, não foi surpresa quea disposição de esgotos em fossas apresentassecaráter protetor em relação à disposição dos excretas em redes coletoras que, quando precariamenteconstruídas ou mantidas, podem facilitar a ocorrência devazamentos, constituindo um risco para as helmintosesintestinais.

Emboraadisposição dosesgotosnos córregostambém tenhatido caráter protetor $(O R=0,370)$ para as helmintoses intestinais, pois afasta rapidamente os excretas da família geradora, esta solução coloca em risco a saúdee o bem-estar das populaçõesquemoram ajusantedo ponto delançamento dosesgotos, além de poluir com carga orgânica os cursos d'água. Assim, obviamente a disposição dosesgotos nos córregos constitui uma solução ambientalmenteinadequada para a disposição dos excretas.

Em resumo, a disposição deesgotos em algum tipo defossa constitui proteção, enquanto, por outro lado, adisposição de excretas a céu aberto - no terreno e nas ruas - constitui um risco para as helmintosesintestinais.

\section{CONCLUSÕES}

Considerando a elevada prevalência encontrada para as hel mintoses intestinais $(21,38 \%)$, recomenda-sequeos programas voltados para o seu controle sejam intensificados nas áreas de assentamento subnormal deJ uiz deFora- M G . Sugere-seatenção especial à saúde de criançascom idadeentredois anos completos e cinco anos incompletos e/ou com rendafamiliar abaixo de dois saláriosmínimos. 0 estudo indicaaindaque, para 0 controledashelmintosesintestinaissão imprescindíveisboascoberturaequalidadenos serviços deabastecimento deáguado siste mapúblico eaeliminação dadisposição dos esgotosnosterrenosou nasruas por meio da implantação deredescoletoras deesgotosou outrassoluçõesalternativasadequadassanitária eambientalmente- em acordo com a tecnologiadisponível na região. 
Por fim, enfatiza-sea necessidade de seprosseguir com novas pesquisasvisando aprofundar a compreensão dosfatores derisco associados às helmintosesintestinaisem assentamentossubnormais, visando aumentar a eficácia das medidas de controledestasenfermidades.

\section{REFERÊNCIAS}

AWWA - AMERICAN WATER WORKS ASSO CIATION. Water quality and treatment. $5^{\text {th }}$ ed. N ew York. M cG raw Hill Inc., 1999.

AZEVEDO, E. de A. Exclusão sanitária em Belo H orizonte - M G: caracterização e associação com indicadores de saúde. Belo $\mathrm{H}$ orizonte. Escola de Engenharia da UFM G, 175p. (D issertação, M estrado em Saneamento, M eio Ambiente e Recursos $\mathrm{H}$ ídricos - área de concentração: Saneamento). 2003.

FEACH EM , R.G .; et. al. Sanitation and disease: health aspects of excreta and wastewater management. Chinchester. John Wiley \& Sons, 501p. 1983.

FIBGE - FUNDAÇÃO INSTITUTO BRASILEIRO DE GEOGRAFIA E ESTATÍSTI$C A$. Censo demográfico: resultado do universo relativo às características da população e do domićlio. Rio de Janeiro. FIBGE, 1991.
HELLER, L.; CASSEB, M.M.S. Abastecimento de água. In: BARROS, R.T. de V. et al; $M$ anual de Saneamento e Proteção Ambiental para os M unicípios, v.2. Belo H orizonte. Escola de Engenharia da UFM G, 1 p. 63-112. 1995.

HOFFM AN N, W.A.; PONS, J.A.; JANER, J.L. The sedimentation concentration method in schistosomiasis mansoni. Puerto Rico Journal of Public Health Tropical M edicine, v.9, p.283291, 1934.

MARA, D.D.; FEACHEM, R.G. Water- and excreta-related diseases: unitary environmental classification. Journal of Environmental Engineering, v.125, n.4, p.334-339, 1999.

MORAES, L.R.S. Health impact of sewerage and drainage in poor urban áreas in Salvador, Brasil. London. London School of $\mathrm{H}$ ygiene and Tropical M edicine of University of London, 243p. (Thesis, D octor of Philosophy in the Faculty of M edicine). 1996.

M ORAES, L.R.S. Avaliação do impacto sobre a saúde das ações de saneamento ambiental em áreas pauperizadas de Salvador - Projeto AISAM . In: H ELLER, L. et al.; $(0 \mathrm{rg}$.) Saneamento e Saúde em Países em D esenvolvimento. Rio de Janeiro. CC\&P Editores, p.281-305. 1997.

PJF - PREFEITURA DE JUIZ DE FORA. JF Bairros. Juiz de Fora. Prefeitura de Juiz de Fora, 2001.
STEPHEN SO N, L.S. The impact of helminth infections on human nutricion. London. Taylor \& Francis, 1987

von SPERLING, M.; COSTA, A.M.L.M. da; CASTRO, A. de A. Esgotos sanitários. In: BARROS, R.T. de V. et al; M anual de Saneamento e Proteção Ambiental para os M unić́ípios, v.2. Belo H orizonte. Escola de Engenharia da UFM G p.113-160. 1995.

WHO - WORLD HEALTH ORGANIZATION. Environmental health in urban-development. Geneve. WHO, 1991

WHO - WORLD HEALTH ORGANIZATION. The world health report - 1997. Geneve. WH O, 1997.

\section{Endereço para correspondência:}

Júlio César Teixeira

Rua Antônio M arinho Saraiva, 115

Apt $^{\circ} 202$ - Dom Bosco

36025-555 - Juíz de Fora - M G -

Brasil

Tel: (32) 3232-6342

E-mail: juliotei@ terra.com.br

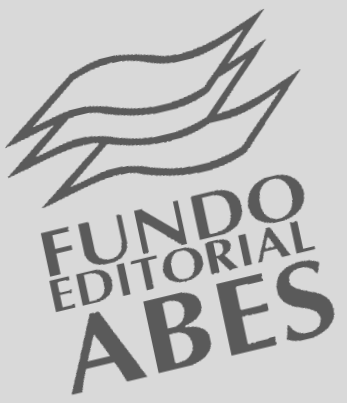

LOJA DE LIVROS ESPECIALIZADA EM SANEAMENTO E MEIO AMBIENTE

\title{
O Fundo Editorial dispõe de mais de 150 títulos,
} mantendo-se sempre atualizado com pesquisas diárias de novos títulos em editoras e universidades.

\author{
Acesse 0 site: www.abes-dn.org.br
}

